\title{
\begin{tabular}{l|l} 
Mitraries & DSpace@MIT
\end{tabular}
}

\author{
MIT Open Access Articles
}

\section{New plasma measurements with a multichannel millimeter- wave fluctuation diagnostic system in the DIII-D tokamak}

The MIT Faculty has made this article openly available. Please share how this access benefits you. Your story matters.

Citation: Hillesheim, J. C. et al. “New plasma measurements with a multichannel millimeterwave fluctuation diagnostic system in the DIII-D tokamak (invited)." Review of Scientific Instruments 81 (2010): 10D907. (c) 2010 American Institute of Physics

As Published: http://dx.doi.org/10.1063/1.3466900

Persistent URL: http://hdl.handle.net/1721.1/66122

Version: Final published version: final published article, as it appeared in a journal, conference proceedings, or other formally published context

Terms of Use: Article is made available in accordance with the publisher's policy and may be subject to US copyright law. Please refer to the publisher's site for terms of use. 


\title{
New plasma measurements with a multichannel millimeter-wave fluctuation diagnostic system in the DIII-D tokamak (invited) ${ }^{a}$
}

\author{
J. C. Hillesheim, ${ }^{1, b)}$ W. A. Peebles, ${ }^{1}$ T. L. Rhodes, ${ }^{1}$ L. Schmitz, ${ }^{1}$ A. E. White, ${ }^{2}$ \\ and T. A. Carter ${ }^{1}$ \\ ${ }^{1}$ Department of Physics and Astronomy, University of California, Los Angeles, Los Angeles, \\ California 90095-1547, USA \\ ${ }^{2}$ Massachusetts Institute of Technology, Cambridge, Massachusetts 02139-4307, USA
}

(Presented 18 May 2010; received 14 May 2010; accepted 12 June 2010; published online 6 October 2010)

\begin{abstract}
A novel multichannel, tunable Doppler backscattering (DBS)/reflectometry system has recently been developed and applied to a variety of DIII-D plasmas. Either DBS or reflectometry can be easily configured for use in a wide range of plasma conditions using a flexible quasi-optical antenna system. The multiple closely spaced channels, when combined with other fluctuation diagnostic systems, have opened up new measurements of plasma properties. For example, the toroidal and fine-scale radial structure of coherent plasma oscillations, such as geodesic acoustic modes, have been probed simultaneously in the core of high temperature plasmas by applying correlation analysis between two toroidally separated DBS systems, as well as within the multichannel array. When configured as a reflectometer, cross-correlation with electron cyclotron emission radiometry has uncovered detailed information regarding the crossphase relationship between density and temperature fluctuations. The density-temperature crossphase measurement yields insight into the physics of tokamak turbulence at a fundamental level that can be directly compared with predictions from nonlinear gyrokinetic simulations. (C) 2010 American Institute of Physics.

[doi:10.1063/1.3466900]
\end{abstract}

\section{INTRODUCTION}

Advances in plasma measurements, in conjunction with comparison to advanced simulations, are critical to achieving validated, predictive capability for the behavior of hightemperature plasmas. Advances in understanding the measurements made with existing diagnostic systems allow new approaches to be applied. Doppler backscattering (DBS), ${ }^{1-7}$ reflectometry, ${ }^{8}$ and electron cyclotron emission (ECE) radiometry $^{9-12}$ are three established millimeter-wave plasma diagnostic techniques. The DIII-D tokamak ${ }^{13}$ currently has multichannel systems of each installed, opening up possibilities for novel measurements through correlation of various local, fluctuating plasma quantities. In this article, multichannel DBS measurements of local coherent mode properties and coupled reflectometer-ECE radiometer measurements of local turbulence properties are presented. An essential element of these measurements is a DBS/reflectometry system with multiple closely spaced channels, allowing measurements with subcentimeter radial resolution. ${ }^{7}$ This capability has also been employed for detailed turbulence studies. ${ }^{14}$

In Sec. II, an overview of the diagnostic hardware is given. In Sec. III, using the phase of a DBS system for measurements is developed. In Sec. IV, the DBS phase analysis approach is applied to a coherent mode, the geodesic acous-

\footnotetext{
anvited paper, published as part of the Proceedings of the 18th Topical Conference on High-Temperature Plasma Diagnostics, Wildwood, New Jersey, May 2010.

b)Electronic mail: jchillesheim@ @ physics.ucla.edu.
}

tic mode (GAM). In Sec. V, coupled reflectometer-ECE radiometer measurements are employed to measure a local turbulence property, the crossphase angle between density, and temperature fluctuations. Finally, conclusions and discussion are included in Sec. VI

\section{MILLIMETER-WAVE HARDWARE AND QUASI-OPTICAL ANTENNA SYSTEM}

The diagnostic employed as part of both sets of measurements in this article is a flexible multichannel millimeterwave system that can be configured for either DBS or reflectometry. A regularly spaced array of five frequencies separated by $350 \mathrm{MHz}$ is launched into the plasma and detected with a set of quadrature mixers (an additional channel was added since the detailed description in Hillesheim et $a l^{7}{ }^{7}$. The dense array of five frequencies can be tuned in the range of 53-78 GHz. A new control scheme has also been implemented. A microcontroller is located near the millimeter-wave hardware, which produces digital outputs that are amplified, filtered, and used as the control voltages for the voltage-controlled oscillator sources. The microcontroller receives commands via a fiber optic Ethernet link from a remote computer. The voltage can be changed without personnel requiring direct access to the millimeter-wave hardware, making changes between shots simple and also allowing changes between settings during a plasma discharge to be programed.

In subsequent sections, two different DBS systems and a correlation ECE (CECE) radiometer ${ }^{12}$ are used in various 
configurations, with the DBS systems located at two different toroidal locations. For concreteness, terminology will be explicitly specified here. The two systems that can be used for either DBS or reflectometry will be referred to as Doppler backscattering or DBS systems except when the beam is purposefully aligned to be normally incident to flux surfaces, when they will be called reflectometers. For brevity, the twochannel system ${ }^{5}$ will be referred as "DBS-2." The fivechannel system ${ }^{7}$ will be referred to as "DBS-5." The abbreviation "ECE" is used to refer to individual channels of the CECE diagnostic. The quasi-optical antenna systems at both diagnostic port locations have been described in detail in previous publications. ${ }^{15,16}$ Both antenna systems are similar, using a collimating lens to direct each beam into a flat mirror, which reflects the beams onto remotely adjustable parabolic mirrors that focus the beams in the plasma. Both systems are monostatic, producing Gaussian beams with beam waists $\left(1 / e^{2}\right.$ power diameter) of $\sim 3-5 \mathrm{~cm}$, depending on the launch frequency. The beam waists are typically $\sim 4-5 \mathrm{~cm}$ for reflectometry or DBS frequencies and $\sim 3 \mathrm{~cm}$ for CECE frequencies. The two port locations are separated by $180^{\circ}$ toroidally and will hereafter be referred as the " $60^{\circ}$ port" and the " $240^{\circ}$ port." The major difference between the two port locations is that the $240^{\circ}$ port mirror can be set to view angles either above or below the horizontal, while the $60^{\circ}$ port can access only angles above the horizontal. Both systems probe near the midplane, so for a plasma vertically centered in the tokamak, measurements either above or below midplane at the $240^{\circ}$ port can be made, while the $60^{\circ}$ port is restricted to probing locations above the midplane. The ray tracing approach described previously ${ }^{7}$ is used with measured port geometry to deduce DBS scattering location and wavenumber information. The same approach has been used between plasma discharges to optimize the mirror angle for normal incidence when reflectometry measurements are desired and to adjust frequency settings to radially align reflectometer and CECE channels.

\section{MODEL EQUATION FOR DBS}

For DBS, a millimeter-wave beam is launched at a frequency that approaches a cutoff in the plasma and at an angle that is oblique to the cutoff surface. This creates a localized scattering region near the cutoff, mostly due to the change in index of refraction along the beam path, where density fluctuations matching the Bragg condition can cause $180^{\circ}$ backscattering of the incident radiation. The backscattered radiation is Doppler shifted by the laboratory frame velocity of the scattering fluctuations. The returning beam is then detected with quadrature mixers. DBS is qualitatively differentiated from conventional reflectometry by the detection of the backscattered signal instead of reflected beam, which does not return to the launch location for DBS configurations.

In previous work, the complex quadrature DBS signal has most commonly been analyzed to yield information about the equilibrium $v_{E \times B}$ flow and its shear. ${ }^{3,17-19}$ The amplitude and the phase of the backscattered electric field can also be analyzed individually. Similar to the method de- scribed below, a finite difference approach has been applied to the DBS phase in previous work. ${ }^{20}$ The amplitude contains information about the relative level of density fluctuations, over a weighted wavenumber range, and factors in the scattering efficiency with its multiple dependencies. ${ }^{21}$ The phase contains information about the equilibrium flow, coherent flow oscillations, turbulent flow fluctuations, and the effect of any optical path length variations. Analysis of the amplitude and phase signals directly allows the additional timewindowing and fast Fourier transform (FFT) procedures necessary to analyze the quadrature signal to be side-stepped; the full time resolution of the diagnostic is then available. By not taking finite differences of the phase the possible introduction of numerical artifacts is avoided. In this section a heuristic model equation for the DBS phase will be described, which can be used to guide data analysis and interpret results.

The "DBS phase," $\varphi_{\mathrm{DBS}}(t)$, to be discussed is the difference in phase between the electric field of the backscattered beam, and the phase of a reference local oscillator. This difference depends on the optical path length and the Doppler shift acquired by the backscattered beam in the scattering process, both of which, in general, can be functions of time

$$
\varphi_{\mathrm{DBS}}(t)=\int_{t_{0}}^{t} \omega_{\mathrm{Dop}}(t) d t+2 \int_{0}^{x_{c}(t)} k(x, t) d x .
$$

The Doppler shift, $\omega_{\text {Dop }}(t)$, is integrated from a reference time $t_{0}$ to time $t$. Gaussian beam and geometry effects are neglected to highlight the dominant physical contributions from the plasma. Considering the beam as an optical ray, the second integral is taken along the propagation path from the antenna at $x=0$, to the cutoff at $x=x_{c}(t)$. Ideally for DBS, the second term is small and can be neglected. Ideally for reflectometry, the beam is aligned normal to the cutoff surface and the first term can be neglected. In reality, both are present to some extent.

These expressions can be further expanded by taking an ansatz for the local velocity of the turbulence. For the propagation velocity of the turbulent structure responsible for the scattering process, assume that there are contributions from the equilibrium $E \times B$ flow, $v_{E \times B}$; from an oscillating coherent mode $v_{m}$, with frequency $\omega_{m}$; and from the turbulent flows $\widetilde{v}$. In principle, the scattering turbulent structure can be advected or convected by any larger scale structures in the plasma, so that there is actually a sum over all scales larger than the scattering structure. For a scattering fluctuation with wavenumber $k_{\perp}$, the plasma frame velocity of each structure, $v_{k}$, then contributes

$$
\widetilde{v}=\sum_{k \leq k_{\perp}} v_{k} .
$$

This situation is pointed out to make clear the difficulty of attempting to measure the plasma frame velocity of the turbulence. Including the above terms, the laboratory frame velocity $v_{\text {Lab }}$ is

$$
v_{\text {Lab }}=v_{E \times B}+v_{m} \cos \left(\omega_{m} t\right)+\widetilde{v} .
$$

The Doppler shift is then $\omega_{\text {Dop }}(t)=k_{\perp} v_{\text {Lab. }}$. (Note that, due to the vertical scattering plane and sheared magnetic field, one 
expects a wavenumber mismatch between the turbulence and the beam that results in a projection of the measured flow from the binormal $E \times B$ direction to the poloidal direction. In the core of standard large aspect ratio tokamaks this is a small effect.) An expression for the phase can then be written down that separates the different physical contributions to $\varphi_{\mathrm{DBS}}(t)$ into multiple terms

$\varphi_{\mathrm{DBS}}(t)=k_{\perp} v_{E \times B} t+\frac{k_{\perp} v_{m}}{\omega_{m}} \sin \left(\omega_{m} t\right)+\widetilde{\varphi}(t)+2 \int_{0}^{x_{c}(t)} k(x, t) d x$.

Depending on the phenomena under investigation, one can then apply appropriate analysis techniques to isolate the desired term. The first term is due to the equilibrium radial electric field and can be extracted most easily by analysis of the complex quadrature signal; although, through smoothing and by taking a derivative, it can be extracted from $\varphi_{\mathrm{DBS}}(t)$. The second term is due to any oscillatory flows in the plasma (in principle, there may of course be multiple coherent modes) and can be examined through spectral analysis of $\varphi_{\text {DBS }}(t)$. The third term is the turbulent flow contribution. In addition to optical path length variations such as islands in the beam path, also accounted for in the fourth term is any backscattering that occurs before the cutoff is reached. This can occur in regions with steep density gradients, or if the beam path is not far above cutoff. These types of effects can occur for any microwave or millimeter-wave diagnostics. With DBS, when there is a significant Doppler shift of the cutoff-localized contribution, this fourth term, if significant, can be filtered out in postprocessing of the complex quadrature signal, since it is centered near zero frequency when it is visible in the data. For reflectometry cases, the fourth term dominates and is essentially due to low- $k$ movements of the cutoff surface; analytical expressions exist ${ }^{8}$ for cases where the other terms can be neglected. For DBS configurations, this term is much smaller than the Doppler shift terms.

It is illuminating to take the Fourier transform of Eq. (4). Assuming the linear term is zero for times less than zero, and absorbing the path length term into $\widetilde{\varphi}(t)$, one finds

$$
\begin{aligned}
\varphi_{\mathrm{DBS}}(\omega)= & k_{\perp} v_{E \times B}\left[\frac{i \delta^{\prime}(\omega)}{4 \pi}-\frac{1}{4 \pi^{2} \omega^{2}}\right] \\
& +\frac{i k_{\perp} v_{m}}{2 \omega_{m}}\left[\delta\left(\omega+\omega_{m}\right)-\delta\left(\omega-\omega_{m}\right)\right]+\widetilde{\varphi}(\omega) .
\end{aligned}
$$

Here $\delta(\omega)$ is the Dirac delta function and $\delta^{\prime}(\omega)$ is its derivative. From Eq. (5) one can extract two expectations for the experimental $\varphi_{\text {DBS }}$ spectrum. From the second term in the first set of parentheses, there should be a $f^{-2}$ component in the spectrum due to the equilibrium component of the $E \times B$ flow. From a coherent mode, one expects a peak at the mode frequency due to the delta functions. These effects can be seen in Fig. 1, where the $f^{-2}$ character is predominant for much of the DBS phase spectrum, except for the peak at the frequency of a coherent mode, falling off at high frequencies. This $f^{-2}$ spectrum is expected and has a physically meaningful origin, in contrast to the $f^{-2}$ spectrum that can occur in reflectometry due to random phase jumps. ${ }^{8,22}$ This $f^{-2}$ spec-

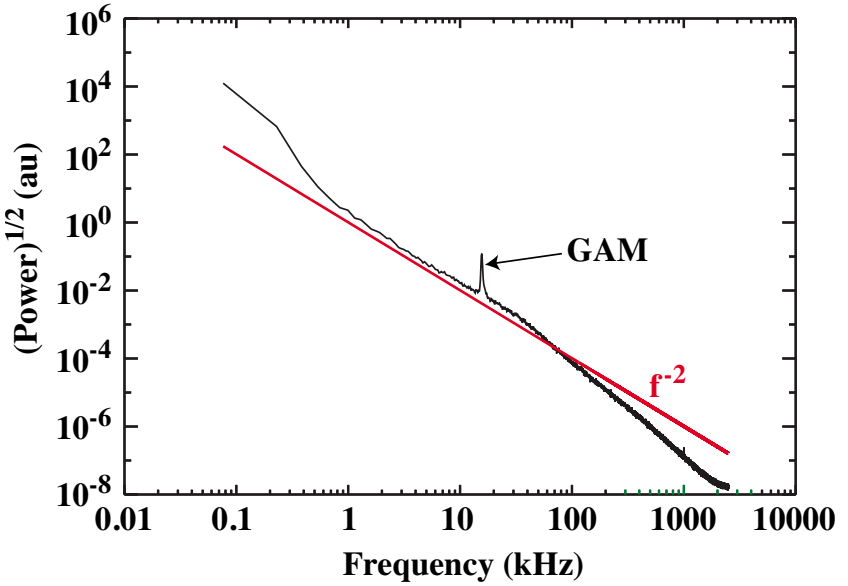

FIG. 1. (Color online) Plotted in black is the DBS phase power spectrum, ensemble-averaged over $500 \mathrm{~ms}$, of a steady-state $L$-mode plasma. The spectrum primarily goes as $f^{-2}$, except for a coherent mode that is present (a GAM in this case), then rolls over at high frequencies where various effects compete.

trum in Fig. 1 is also qualitatively different from the reflectometer spectra, which exist over the entire dynamic range of the diagnostic, whereas the $f^{-2}$ of $\varphi_{\mathrm{DBS}}$ falls off for time scales much faster than equilibrium time scales. This occurs since the equilibrium $v_{E \times B}$ flow is assumed constant in the ansatz, when in reality it is only constant in a statistical sense. There also a significant amount of power at low frequencies, which could be due to zonal flow activity.

\section{MULTICHANNEL CORRELATION ANALYSIS OF COHERENT PLASMA MODES}

Here, we present an example of using correlations between DBS channels to measure the toroidal and fine-scale radial structure of flow oscillations associated with a coherent plasma mode; in this case, the GAM. ${ }^{23}$ DBS has previously been used to study the GAM. ${ }^{20,24}$ Standard correlation methods are applied, with statistical estimates of the standard deviation used for error bars. ${ }^{25}$ As can be seen from Eq. (5), spectral analysis of the DBS phase can be used to identify and study the mode. The same data have also been analyzed using the complex quadrature signal by finding the mean frequency in short time windows, then Fourier analyzing a time series of such calculations. The same results are attained, but with much worse statistics due to taking FFTs of the data twice. The potential structure associated with the electrostatic GAM is expected to be dominated by an axisymmetric (toroidal mode number $n=0$, poloidal mode number $m=0$ ) flow, with a finite radial wavenumber and, for typical tokamak conditions, outward radial propagation. ${ }^{26-28}$

In the high safety factor, circular plasma, electrostatic limit, the GAM frequency becomes $\omega_{\mathrm{GAM}}=\sqrt{2}\left(c_{s} / R\right)$. The sound speed is $c_{s}=\sqrt{\left(T_{e}+\gamma_{i} T_{i}\right) / m_{i}}$, with kinetic calculations yielding $\gamma_{i}=7 / 4$ for the GAM. ${ }^{29,30}$ Here, $R$ is the major radius, $T_{e}$ is the electron temperature, $T_{i}$ is the ion temperature, and $m_{i}$ is the ion mass. The GAM damping rate is proportional to $e^{-q^{2}}$, where $q$ is the safety factor, limiting its existence to the plasma edge in most cases. ${ }^{30}$ Figure 2 shows a spectrogram of the DBS phase and the electron temperature 


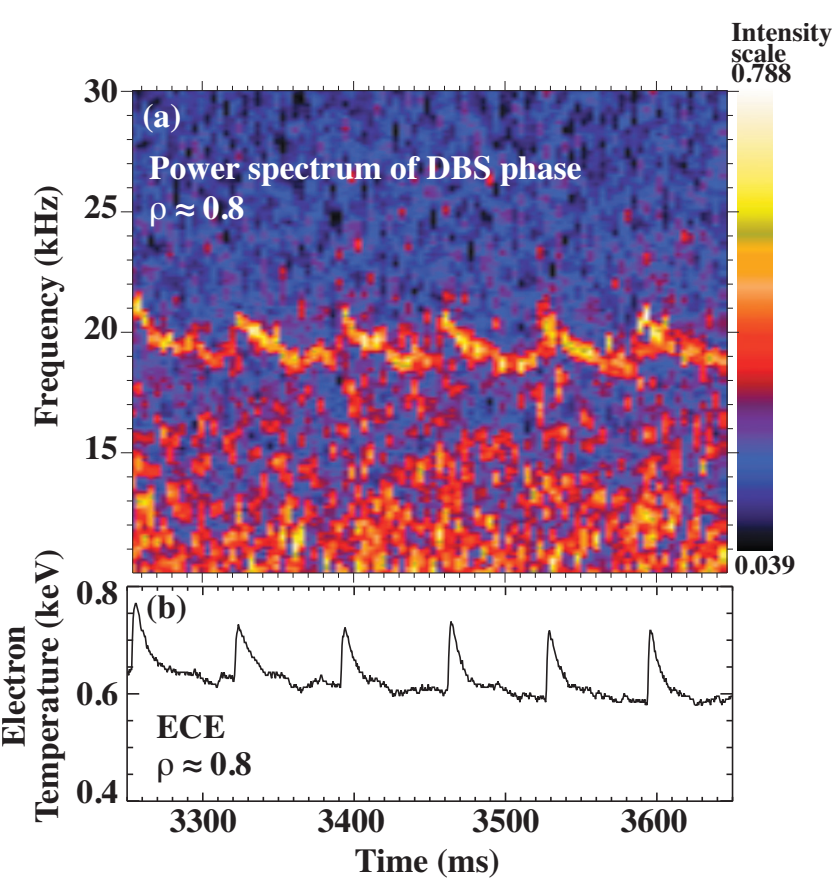

FIG. 2. (Color) (a) Spectrogram of the DBS phase at $\rho \approx 0.8$, showing a flow oscillation from a GAM as the GAM's frequency is modulated by sawtooth heat pulses. (b) Electron temperature from ECE at the same radial location.

from a profile ECE diagnostic ${ }^{31}$ at nearly the same radial location in a neutral beam heated $L$-mode DIII-D discharge. There is nothing significant on the fast magnetic probes or in spectral analysis of the ECE at this frequency, so the DBS phase oscillation can be identified through Eq. (5) as due to local turbulence flow. Calculation of the GAM frequency using local plasma parameters produces about $20 \mathrm{kHz}$. The clear relationship between the electron temperature pulses from sawtooth crashes and flow oscillation identifies the mode as a GAM.

Correlation analysis can be applied between the multiple DBS channels to extract more information about the GAM. The data in this section were acquired with DBS- 5 at the $60^{\circ}$ port and DBS- 2 at the $240^{\circ}$ port. Figure 3 shows the ensemble averaged coherency and crossphase, $\alpha_{v_{\mathrm{GAM}}}$, between the phase signal, $\varphi_{\mathrm{DBS}}$, of two DBS channels at locations separated by $180^{\circ}$ toroidally, but aligned within $1 \mathrm{~cm}$ radially at $\rho \approx 0.8$, where $\rho$ is the square root of the normalized toroidal flux. The safety factor at the measurement location is about 2, lower than most previous observations, which have mostly been acquired at higher $q$, near the last closed flux surface. The width of the coherent peak is due mostly to the sawtooth oscillations. The high coherence at low frequencies is due primarily to the equilibrium component of the radial electric field. The channels are also offset poloidally by about $5^{\circ}$ due to port geometries, with DBS-5 probing slightly above the midplane and DBS- 2 slightly below. The small phase difference between the two channels is consistent with expected axisymmetric $(m=0, n=0)$ flow structure for the GAM.

Figure 4 shows the crossphase among the seven DBS channels, using both DBS-5 and DBS-2, at the frequency of maximum coherency for the GAM, referenced to the middle

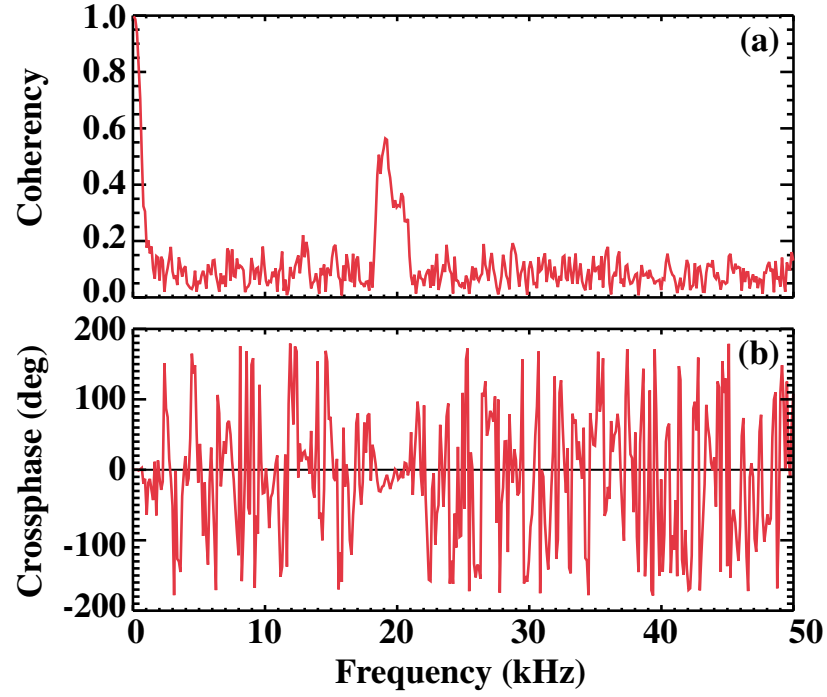

FIG. 3. (Color online) (a) Coherency of the DBS phase between two DBS channels at toroidal locations separated by $180^{\circ}$, radially aligned at $\rho \approx 0.8$, and ensemble averaged over $400 \mathrm{~ms}$. (b) Crossphase between the same two channels.

channel of DBS-5. The radial separation of the DBS-5 channels in Fig. 4 is $\sim 0.5 \mathrm{~cm}$. Assuming that the GAM is dominantly axisymmetric $(m=0, n=0)$, which is consistent with Fig. 3, then, even though toroidally and poloidally separated channels are used, the change in crossphase is due to the difference in radial location. The ray tracing code GENRAY, ${ }^{32}$ using experimental equilibrium and density profiles, is used to determine the locations. The crossphase $\alpha_{v_{\mathrm{GAM}}}(\rho)$ between the channels shows a linear increase outward, consistent with outward radial propagation-for a standing wave one would expect $0^{\circ}$ or $180^{\circ}$. Applying a linear fit to the data, a radial wavelength of $\lambda_{\mathrm{GAM}} \approx 3 \mathrm{~cm}$ is calculated. These observations are consistent with previous experiments on DIII-D (Refs. 33 and 34) and with experiments on other tokamaks. ${ }^{35,36}$ The error bars are purely statistical and do not account for systematic errors. The $\rho \approx 0.80$ point from the $240^{\circ}$ port appears to be inconsistent with the fit. This could in part be due to a low signal level for that channel. When

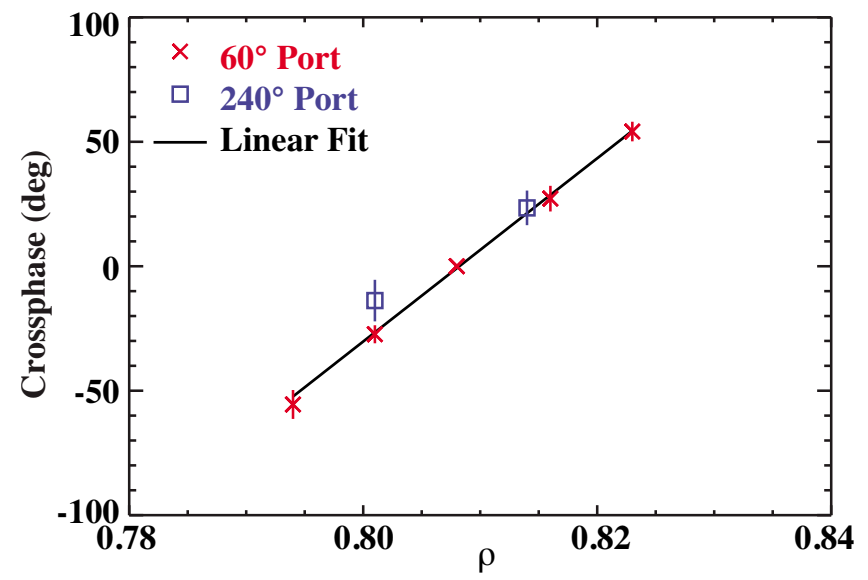

FIG. 4. (Color online) The crossphase, $\alpha_{v_{\mathrm{GAM}}}(\rho)$, among the phase of seven DBS channels at the GAM frequency plotted against $\rho$, ensemble averaged over $400 \mathrm{~ms}$, showing outward radial propagation of the GAM. The center channel of DBS-5 is the reference. 
there is a low signal level from the plasma, the backscattering signal competes with system noise and ambient pickup, potentially decreasing the coherency for incoherent noise and biasing the crossphase toward zero for ambient pickup. A second explanation is that the radial wavenumber for the GAM is expected to depend on poloidal angle $;^{27}$ the two explanations cannot be distinguished with this data set.

\section{MULTIFIELD CORRELATION ANALYSIS OF PLASMA TURBULENCE}

In this section we present measurements of the crossphase between electron density and electron temperature fluctuations, $\alpha_{n_{e}}, T_{e}$, made by arranging DBS-5 as a reflectometer and correlating its channels with ECE data from a radiometer-based CECE diagnostic. ${ }^{12}$ The highest coherency between each pair of channels from the coupled reflectometer-ECE system is used in the results presented. The coherency is observed to decrease within the DBS-5 array for a given ECE channel; this shows how indispensable the dense array of channels is for successfully obtaining and localizing the measurement. Previous literature has focused on using the reflectometer phase to reconstruct attributes of plasma turbulence, such as spectrum shapes and fluctuation levels. Issues that can arise when analyzing the reflectometer phase have been pointed out, with large aperture optical imaging systems suggested as the solution. ${ }^{37}$ Past efforts to measure $\alpha_{n_{e}, T_{e}}$ also used the reflectometer phase. ${ }^{38}$ An alternative approach that has received less attention is to use the reflectometer amplitude signal. ${ }^{16}$ Although an analytical treatment of the amplitude is challenging, it has been demonstrated in both experiment ${ }^{39,40}$ and in two-dimensional full wave simulations ${ }^{41}$ that in many cases the homodyne or amplitude signals can be a better proxy for density fluctuations at the cutoff than the phase.

The following measurements were obtained in an Ohmic plasma discharge with electron cyclotron heating $(\mathrm{ECH})$ in the DIII-D tokamak. The DBS-5 and CECE diagnostics were diplexed together and shared the same quasi-optical antenna system at the $60^{\circ}$ port. Both diagnostics are sensitive to low- $k$ fluctuations $\left(k_{\perp} \rho_{i} \leq 0.5\right)$. DBS-5 was aligned for reflectometry, and both the multiple reflectometry channels and adjustable channels from the CECE diagnostic were tuned to probe the same volume of plasma, at $\rho \approx 0.6$. By doing this, either the phase or the amplitude of the reflectometer channels can be correlated with the ECE channels. The plasma was optically thick at the measurement location, so no contribution of density fluctuations to the ECE signals would be expected. Figure 5 compares the coherency and crossphase attained by correlating each reflectometer signal with an ECE channel. Note that the absolute value of the coherency is lowered by the thermal noise contribution to the ECE signal. Both reflectometer signal types show coherency, although the coherency with the amplitude is higher. This is a representative result-when the reflectometer phase can be recovered and correlates with ECE, the amplitude always does, and with higher coherency. However, the correlation
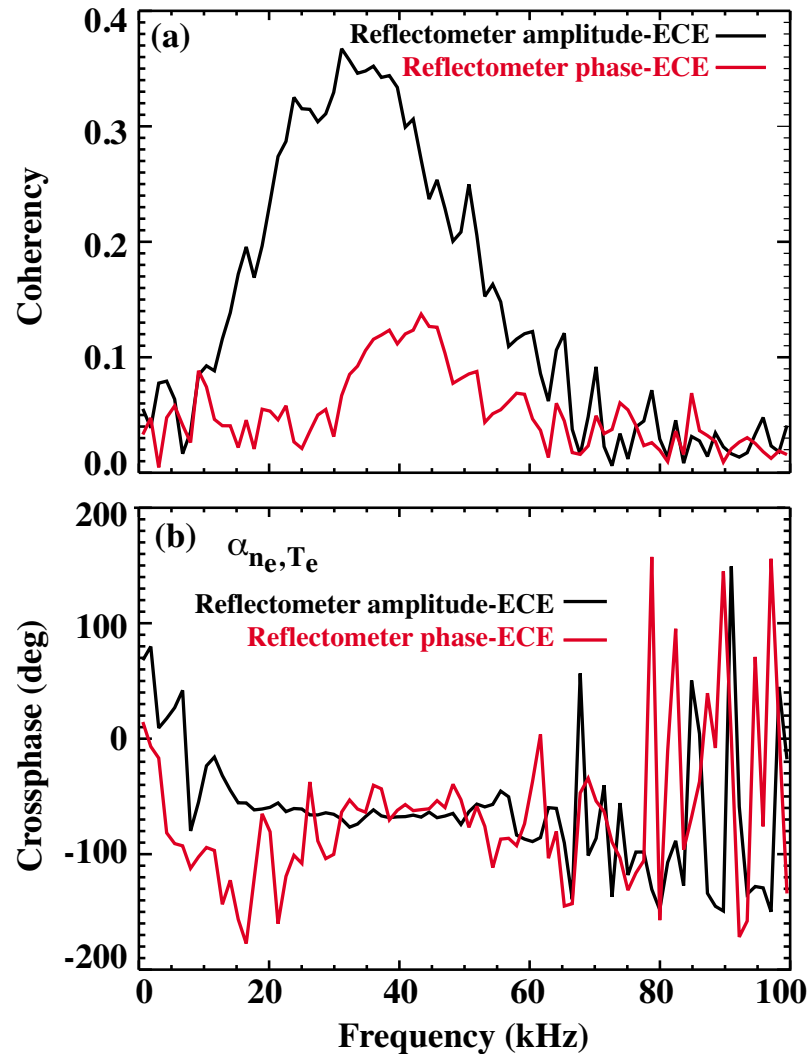

FIG. 5. (Color online) Comparison of cross-correlation of reflectometer phase and ECE data to the reflectometer amplitude from the same channel and the same ECE signal, ensemble averaged over $400 \mathrm{~ms}$ using 975 records. Error bars omitted for clarity. (a) Comparison of the coherency. (b) Comparison of the crossphase angle, $\alpha_{n_{e}, T_{e}}$, using the reflectometer as the reference signal.

with the phase signal is often negligible, even when correlation with the amplitude is present. When both are present for a significant frequency range, the crossphase angle is the same, within statistical error measures. These observations are consistent with the amplitude being a better proxy for measuring local density fluctuations from turbulence.

A sequence of times with different amounts of ECH power occurred in the same discharge. By applying correlation analysis to a steady-state time period during each ECH power step, a clear trend in the crossphase appears, which is shown in Fig. 6. The effect of the ECH is to increase both the electron temperature and electron temperature scale length by roughly equivalent amounts, making the scan primarily consist of concurrent changes to the temperature ratio between ions and electrons and to the collisionality when nondimensional quantities are considered. There are also small changes to the density and density gradient during the scan, the effects of which are not captured in Fig. 6.

An atypical aspect of this discharge was that the plasma was shifted vertically to make reflectometry measurements possible with the port geometry at that time. This made the plasma significantly up-down asymmetric. For this reason and also due to density profiles with undesirable levels of uncertainty, comparison to gyrokinetic simulations would have been challenging. A subsequent experiment, optimized for comparison to nonlinear gyrokinetic simulations, was 


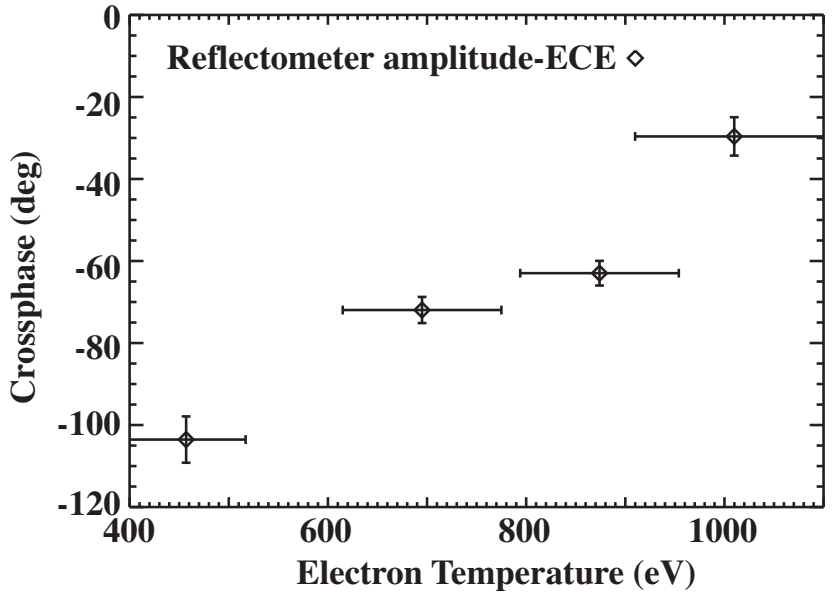

FIG. 6. The changes to the crossphase between electron density and temperature fluctuations, $\alpha_{n_{e}, T_{e}}$, with increasing electron temperature at $\rho \approx 0.6$, calculated with reflectometer amplitude correlated with an ECE channel. The frequency at which the maximum coherency occurs for each pair is used to calculate the crossphase and error bars.

performed. For one condition, quantitative agreement for the crossphase, $\alpha_{n_{e}, T_{e}}$, was found between experimental measurements and the simulation. ${ }^{16}$

\section{CONCLUSION AND DISCUSSION}

In this article, the DBS phase has been analyzed, with presentation of a heuristic model equation and application to measurements of the toroidal and fine-scale radial structure of the GAM. The GAM measurements are consistent with theoretical expectations. Looking forward, the radial wavenumber measurements can be used to test recent theoretical predictions of its scaling and geometric dependencies. ${ }^{26-28}$ The radial wavenumber and amplitude of the GAM determine the radial electric field shear associated with the mode, so understanding the physics of these mode attributes is important for understanding the interaction of the GAM with turbulence. The nonlinear interactions of the GAM with zonal flows and ambient turbulence can also be studied. More generally, the analysis approach presented here can be applied to many classes of plasma instabilities in tokamaks to investigate their structure in detail.

Measurements of the crossphase angle between electron density and electron temperature fluctuations have also been presented. The results show that this quantity, a fundamental characteristic of the instabilities present, can be locally measured in the core of a tokamak. As has been shown, ${ }^{16}$ this measurement can be used for detailed comparison to nonlinear gyrokinetic simulations. This measurement may be able to provide insight into the dominant instability mode in plasma discharges for particular conditions, nonlinear effects such as the influence of $E \times B$ shear on the crossphase, ${ }^{42}$ and concepts such as the importance of damped eigenmodes, ${ }^{43}$ each of which might be able to produce measurable changes.

Together, these advances in measurement capabilities and understanding demonstrate some of the possibilities available when multichannel, multifield fluctuation diagnostic systems are applied in novel ways in a high-temperature plasma.

\section{ACKNOWLEDGMENTS}

This research was supported by U.S. Department of Energy under Grant Nos. DE-FG02-08ER54984 and DE-FG03$01 E R 54615$ and Contract No. DE-FC02-04ER54698. J.C.H.'s work was performed through the Fusion Energy Sciences Fellowship Program administered by Oak Ridge Institute for Science and Education under a contract between the U.S. Department of Energy and the Oak Ridge Associated Universities. The authors would like to thank the entire team at DIII-D for their support of these experiments. The authors would also like to thank C. Wannberg, X. Nguyen, and R. Martin for their technical support.

${ }^{1}$ E. Holzhauer, M. Hirsch, T. Grossmann, B. Brañas, and F. Serra, Plasma Phys. Controlled Fusion 40, 1869 (1998).

${ }^{2}$ M. Hirsch, E. Holzhauer, J. Baldzuhn, B. Kurzan, and B. Scott, Plasma Phys. Controlled Fusion 43, 1641 (2001).

${ }^{3}$ G. D. Conway, J. Schirmer, S. Klenge, W. Suttrop, E. Holzhauer, and the ASDEX Upgrade Team, Plasma Phys. Controlled Fusion 46, 951 (2004).

${ }^{4}$ P. Hennequin, C. Honoré, A. Truc, A. Quéméneur, N. Lemoine, J. Chareau, and R. Sabot, Rev. Sci. Instrum. 75, 3881 (2004).

${ }^{5}$ L. Schmitz, G. Wang, J. C. Hillesheim, T. L. Rhodes, W. A. Peebles, A. E. White, L. Zeng, T. A. Carter, and W. Solomon, Rev. Sci. Instrum. 79, 10F113 (2008).

${ }^{6}$ T. Happel, T. Estrada, E. Blanco, V. Tribaldos, A. Cappa, and A. Bustos, Rev. Sci. Instrum. 80, 073502 (2009).

${ }^{7}$ J. C. Hillesheim, W. A. Peebles, T. L. Rhodes, L. Schmitz, T. A. Carter, P.-A. Gourdain, and G. Wang, Rev. Sci. Instrum. 80, 083507 (2009).

${ }^{8}$ R. Nazikian, G. J. Kramer, and E. Valeo, Phys. Plasmas 8, 1840 (2001).

${ }^{9}$ S. Sattler and H. J. Hartfuss, Phys. Rev. Lett. 72, 653 (1994).

${ }^{10}$ G. Cima, R. V. Bravenec, A. J. Wootton, T. D. Rempel, R. F. Gandy, C. Watts, and M. Kwon, Phys. Plasmas 2, 720 (1995).

${ }^{11}$ C. Watts, H. J. Hartfuss, and M. Hase, Rev. Sci. Instrum. 75, 3177 (2004).

${ }^{12}$ A. E. White, L. Schmitz, G. R. McKee, C. Holland, W. A. Peebles, T. A. Carter, M. W. Shafer, M. E. Austin, K. H. Burrell, J. Candy, J. C. DeBoo, E. J. Doyle, M. A. Makowski, R. Prater, T. L. Rhodes, G. M. Staebler, G. R. Tynan, R. E. Waltz, and G. Wang, Phys. Plasmas 15, 056116 (2008).

${ }^{13}$ J. Luxon, Nucl. Fusion 42, 614 (2002).

${ }^{14}$ J. C. DeBoo, C. Holland, T. L. Rhodes, L. Schmitz, G. Wang, A. E. White, M. E. Austin, E. J. Doyle, J. Hillesheim, W. A. Peebles, C. C. Petty, Z. Yan, and L. Zeng, Phys. Plasmas 17, 056105 (2010).

${ }^{15}$ L. Schmitz, A. White, G. Wang, J. DeBoo, J. deGrassie, G. McKee, J. Hillesheim, W. Peebles, T. Rhodes, T. Carter, E. Doyle, L. Zeng, K. Burrell, C. Petty, J. Kinsey, W. Solomon, G. Staebler, and the DIII-D Team, Nucl. Fusion 49, 095004 (2009).

${ }^{16}$ A. E. White, W. A. Peebles, T. L. Rhodes, C. H. Holland, G. Wang, L. Schmitz, T. A. Carter, J. C. Hillesheim, E. J. Doyle, L. Zeng, G. R. McKee, G. M. Staebler, R. E. Waltz, J. C. DeBoo, C. C. Petty, and K. H. Burrell, Phys. Plasmas 17, 056103 (2010).

${ }^{17}$ J. Schirmer, G. Conway, H. Zohm, W. Suttrop, and the ASDEX Upgrade Team, Nucl. Fusion 46, S780 (2006).

${ }^{18}$ E. Trier, L. Eriksson, P. Hennequin, C. Fenzi, C. Bourdelle, G. Falchetto, X. Garbet, T. Aniel, F. Clairet, and R. Sabot, Nucl. Fusion 48, 092001 (2008).

${ }^{19}$ T. Estrada, T. Happel, L. Eliseev, D. López-Bruna, E. Ascasíbar, E. Blanco, L. Cupido, J. M. Fontdecaba, C. Hidalgo, R. Jiménez-Gómez, L. Krupnik, M. Liniers, M. E. Manso, K. J. McCarthy, F. Medina, A. Melnikov, B. van Milligen, M. A. Ochando, I. Pastor, M. A. Pedrosa, F. L. Tabarés, D. Tafalla, and TJ-II Team, Plasma Phys. Controlled Fusion 51, 124015 (2009).

${ }^{20}$ G. D. Conway, B. Scott, J. Schirmer, M. Reich, A. Kendl, and the ASDEX Upgrade Team, Plasma Phys. Controlled Fusion 47, 1165 (2005).

${ }^{21}$ C. Lechte, IEEE Trans. Plasma Sci. 37, 1099 (2009).

${ }^{22}$ R. Nazikian and E. Mazzucato, Rev. Sci. Instrum. 66, 392 (1995).

${ }^{23}$ N. Winsor, J. L. Johnson, and J. M. Dawson, Phys. Fluids 11, 2448 (1968).

${ }^{24}$ G. D. Conway, C. Tröster, B. Scott, K. Hallatschek, and the ASDEX Upgrade Team, Plasma Phys. Controlled Fusion 50, 055009 (2008).

${ }^{25}$ J. S. Bendat and A. G. Piersol, Random Data, 3rd ed. (Wiley, New York, 2000). 
${ }^{26}$ C. Nguyen, X. Garbet, and A. I. Smolyakov, Phys. Plasmas 15, 112502 (2008).

${ }^{27}$ R. Hager and K. Hallatschek, Phys. Plasmas 16, 072503 (2009).

${ }^{28}$ R. Hager and K. Hallatschek, Phys. Plasmas 17, 032112 (2010).

${ }^{29}$ H. Sugama and T. Watanabe, Phys. Plasmas 13, 012501 (2006).

${ }^{30}$ Z. Gao, K. Itoh, H. Sanuki, and J. Q. Dong, Phys. Plasmas 15, 072511 (2008).

${ }^{31}$ M. E. Austin and J. Lohr, Rev. Sci. Instrum. 74, 1457 (2003).

${ }^{32}$ A. P. Smirnov and R. W. Harvey, Bull. Am. Phys. Soc. 40, 1837 (1995).

${ }^{33}$ G. R. McKee, R. J. Fonck, M. Jakubowski, K. H. Burrell, K. Hallatschek, R. A. Moyer, D. L. Rudakov, W. Nevins, G. D. Porter, P. Schoch, and X. Xu, Phys. Plasmas 10, 1712 (2003).

${ }^{34}$ G. R. McKee, D. K. Gupta, R. J. Fonck, D. J. Schlossberg, M. W. Shafer, and P. Gohil, Plasma Phys. Controlled Fusion 48, S123 (2006).

${ }^{35}$ T. Ido, Y. Miura, K. Kamiya, Y. Hamada, K. Hoshino, A. Fujisawa, K. Itoh, S. Itoh, A. Nishizawa, H. Ogawa, Y. Kusama, and JFT-2M group, Plasma Phys. Controlled Fusion 48, S41 (2006).
${ }^{36}$ T. Lan, A. D. Liu, C. X. Yu, L. W. Yan, W. Y. Hong, K. J. Zhao, J. Q. Dong, J. Qian, J. Cheng, D. L. Yu, and Q. W. Yang, Phys. Plasmas 15, 056105 (2008).

${ }^{37}$ E. Mazzucato, Plasma Phys. Controlled Fusion 46, 1271 (2004).

${ }^{38}$ M. Häse, M. Hirsch, and H. J. Hartfuss, Rev. Sci. Instrum. 70, 1014 (1999).

${ }^{39}$ T. L. Rhodes, W. A. Peebles, E. J. Doyle, P. Pribyl, M. Gilmore, R. A. Moyer, and R. D. Lehmer, Plasma Phys. Controlled Fusion 40, 493 (1998).

${ }^{40}$ M. Gilmore, W. A. Peebles, and X. V. Nguyen, Plasma Phys. Controlled Fusion 42, L1 (2000).

${ }^{41}$ E. Blanco, T. Estrada, and T. Happel, Proceedings of the Ninth International Reflectometry Workshop-IRW9, 2009.

${ }^{42}$ P. W. Terry, D. E. Newman, and A. S. Ware, Phys. Rev. Lett. 87, 185001 (2001).

${ }^{43}$ D. R. Hatch, P. W. Terry, W. M. Nevins, and W. Dorland, Phys. Plasmas 16, 022311 (2009). 